\title{
A pre-test post-test assessment of non-invasive keratograph break up time and corneal epithelial thickness after vaping
}

\author{
Alvin Munsamy, Bhavna Bhanprakash, Amina Sirkhot, Lufun Mlambo, Samukelisiwe \\ Dlamuka, Ndumiso Mhlongo, Ronelle Naidoo
}

University of KwaZulu-Natal College of Health Sciences, Optometry.

\begin{abstract}
:
Background: The effects of electronic cigarettes on the ocular surface has yet to be shown. The purpose of the study was to assess the impact of e-cigarette use on the anterior corneal surface integrity.

Methods: Forty three males and 21 females with an average of 21 years were required to vape $0.05 \mathrm{ml}$ of e-liquid of $8 \mathrm{mg}$ nicotine concentration. Corneal epithelial thickness (CET) and Non Invasive Keratograph Tear Break up Time (NIKBUT) measurements were obtained prior to and post vaping. The Optovue iVue optical coherence topographer was used to measure central; superior; inferior; nasal and temporal CET and NIKBUT was assessed using the Oculus Keratograph 5M.

Results: There was a mean increase for central corneal epithelial thickness of 0.3448 microns. The superior CET increased by 0.2414 microns. The inferior CET increased by 0.2931 microns. The nasal CET increased by 0.2069 microns. The temporal CET increased by 0.2759 microns. The mean change in NIKBUT post-vaping was an increase of 1.40 seconds. All observations occurred at $\mathrm{p}>0.05$.

Conclusion: The acute effect of e-cigarette use does not impact corneal epithelial thickness and non-invasive keratography tear break up time after 10 puffs mild exposure but more research is needed to assess if this remains the case with more frequent, higher exposure.
\end{abstract}

Keywords: Pre-test post-test assessment, non-invasive keratograph breakup time, corneal epithelial thickness, vaping. DOI: https://dx.doi.org/10.4314/ahs.v19i4.13

Cite as: Munsamy A, Bhanprakash B, Sirkhot A, Mlambo L, Dlamuka S, Mblongo N, Naidoo R. A pre-test post-test assessment of non-invasive keratograph break up time and corneal epithelial thickness after vaping. Afri Health Sci. 2019;19(4):2926-2933. https:/ I $d x$. doi.org/10.4314/abs.v19i4.13

\section{Introduction}

Cigarette smoking is harmful to users and can have severe effects on the body. According to the World Health Organisation (WHO), tobacco is a legal drug that kills many of its users. ${ }^{1}$ In recent times, many individuals have turned to electronic cigarettes, which do not contain tobacco and is marketed as a safer option on health. Electronic cigarettes, better known as e-cigarettes, are battery-operated devices, which deliver nicotine with flavourings and other chemicals to users in a vapour instead of in smoke. Puffing activates the battery-powered heating device, which vaporizes the liquid in the cartridge. The resulting aerosol or vapour is then inhaled (called "vaping"). ${ }^{2}$

\section{Corresponding author: \\ Alvin Munsamy, \\ University of KwaZulu-Natal College \\ of Health Sciences, Optometry. \\ Email: alvinjmunsamy@gmail.com, munsamya1@ukzn.ac.za}

Electronic cigarettes According to the Food and Drug Administration (FDA), e-cigarettes contain nicotine, propylene glycol, glycerine, tobacco flavouring and other contaminants potentially harmful to humans. ${ }^{3}$ E-cigarettes are designed to deliver nicotine in an aerosolized manner that simulates an authentic smoking experience without the real smoke. ${ }^{3}$ The nicotine concentration of the solutions or cartridges can be purchased in strengths ranging from 0 to $24 \mathrm{mg}$ or more, according to user preference. ${ }^{3}$ A study by Goniewicz et al. ${ }^{4}$ found that the toxicants levels in e-cigarette vapour was much lower when compared to conventional cigarette smoke. A systematic review by Hess et $\mathrm{al}^{5}$ on passive exposure to electronic cigarette vapour found that this vapour may pose a health risk to bystanders. The toxicants in the vapour may have an impact on indoor air quality thus questioning second hand exposure of vapour despite its lower concentrations than cigarette smoking. ${ }^{5}$

Since 2005, the electronic cigarette industry has grown from one manufacturer in China to an estimated three 
billion dollars global business with 466 brands and almost 8,000 different flavours. ${ }^{6}$ There is concern that vaping will serve as a gateway to nicotine addiction and conventional cigarette smoking, predominantly for young people as experimentation is rapidly increasing, with e-cigarette use in this group doubling from 2008 to 2012 in North America, European Union and Republic of Korea. ${ }^{6}$

In 2012, the Medicines and Substances Control Act in South Africa states electronic nicotine delivery systems are scheduled devices and should be purchased at a pharmacy with a doctor's prescription. ${ }^{7}$ Attractive marketing of e-cigarette's non-toxicity and appealing flavouring options are turning many cigarette smokers to vaping, especially adolescents. ${ }^{8}$

Smoking-related diseases are primarily attributed to the toxicants and carcinogens present in the tobacco smoke emitted during the combustion process that is absent in vaping. ${ }^{9}$ Moreover, nicotine present in both conventional cigarettes and electronic cigarettes, is not classified as carcinogenic by the World Health Organisation and has minimal contribution in promoting obstructive lung disease and artherosclerotic heart disease. ${ }^{9}$ Thus, the socioeconomic relief afforded from vaping over cigarette smoking will benefit society by the reduction in health care costs, the sparing of millions of smoking-related deaths worldwide, and the positive environmental impact by reducing the burning of vegetable matter.

Zhang et al..$^{10}$ also indicated that nicotine delivery is highly dependent on a number of factors, including vaping technique, particle evolution, and cloud effects. Trtchounian et al. ${ }^{11}$ determined that aerosol density for e-cigarettes remained fairly constant for the first 10 puffs of a new e-cigarette cartridge. ${ }^{11}$ However, a decrease in aerosol density was observed as each cartridge approached its terminal life of 300 puffs. ${ }^{11}$

Exposure to glycol mist, found in e-cigarette cartridges and solutions, may lead to dry eye. ${ }^{12}$ Dry eye has also been investigated in cigarette smokers and has been shown to have deteriorating effects on the lipid layer of the pre-corneal tear film. ${ }^{13}$

Acute vaping was found to have immediate adverse physiological effects similar to some of the effects observed with smoking systemically but the long-term health effects of vaping are not known. ${ }^{14}$

There is a paucity of literature on the use of e-cigarette on the ocular surface. However the effects of cigarette smoking has been shown to lower tear film break-up time and corneal and conjunctival sensitivity; and delayed corneal epithelial wound healing from keratitis by an average of 23.9 days. ${ }^{15,16}$ Since smoking disrupts the corneal surface, it remains to be shown whether e-cigarettes have a similar effect or none. The focus of this study was to determine the effect of vaping on its outer-most ocular surface, the corneal epithelium with the use of corneal pachymetry and a non-invasive tear stability test.

\section{Methods}

This is a quasi-experimental one group pretest posttest design, that was conducted at the Discipline of Optometry; Eye Clinic; University of KwaZulu-Natal (Westville campus), RSA. Participants were recruited using a purposive sampling technique. The inclusion criteria were participants within the legal smoking age of older than 18 years. Participants with either acute or chronic systemic diseases, history or presence of corneal diseases; ocular or systemic medications; recreational drug users; contact lens users and persons who had undergone corneal surgery were excluded.

The study determined the effect of vaping on corneal epithelial thickness using Optovue iVue optical coherence tomographer (OCT) pachymetry and tear film stability was assessed using Non-Invasive Keratograph Break-up Time (NIKBUT) with the Oculus keratograph 5M. Data collection comprised of both pre-test and post-test measurements.

The Optovue iVue was used to generate a corneal scan producing a pachymetry map showing corneal epithelial thickness for $6 \mathrm{~mm}$ of the cornea in microns. This was further divided into the central, superior, inferior, nasal and temporal zones. The Optovue is validated as a reliable tool for epithelial pachymetry. ${ }^{17}$

The Oculus Keratograph, has 22 placido rings projected onto the cornea, with more than 1,000 measurement points per ring, resulting in 22,000 analyzed data points per frame. The video recording lasts up to a maximum of 25 seconds, or until the patient's next blink, whichever occurs first. ${ }^{18}$

The Oculus Keratograph 5M produced a NIKBUT in seconds utilising the percentage of all tear film segments which were broken up. The average tear film break up time of all broken segments is then calculated. Tear film stability was classified by levels 0,1 and 2 with the aver- 
age break-up value. Level zero represented a stable tear film of greater than or equal to 14 seconds. Level one represented a critical tear film of greater than 7 seconds but less than 14 seconds and level two represented an unstable tear film of less than 7 seconds.

The Oculus Keratograph 5M provided a simple, non-invasive screening test for dry eye with acceptable repeatability and reproducibility and it should be considered as an alternative method in the diagnosis and follow up of patients with dry eye. ${ }^{19}$

NIKBUT was chosen over Tear Thinning Time (TT'T) and Tear Break Up Time (TBUT) as this test provided an electronic in vivo assessment of the tear film stability independent of fluorescein and examiner influence. It also provided a representation of tear break-up over time, including a tear map showing the size and location of the tear-break region, as well as the first break-up time and an average break-up time. ${ }^{20}$ The oculus keratograph, has 22 placido rings projected onto the cornea, with more than 1,000 measurement points per ring, resulting in 22,000 analyzed data points per frame. The video recording lasts up to a maximum of 25 seconds, or until the patient's next blink, whichever occurs first. ${ }^{20}$

A preliminary study consisting of 11 participants was used to determine the amount of e-liquid required to equate to 10 puffs which according to Dawkins et al. ${ }^{21}$ allowed for detection in plasma. Each participant was required to vape 10 puffs of e-liquid of $8 \mathrm{mg}$ nicotine concentration with the e-cigarette being weighed at different intervals on the AS.R series, AS 220.R2 analytical balance scale to ensure that the required amount of e-liquid was vaped as a measure of puffs. This was to allow for the standardisation required for each participant to vape 10 puffs. The preliminary study revealed that on average participants should vape 10 puffs to consume $0.05 \mathrm{ml}$ e-liquid.

Participants were screened prior to admission into the study to ensure that the inclusion criteria were satisfied. As part of the screening process a thorough case history was conducted to rule out systemic pathology and determine the smoking status of the participant. Slit lamp biomicroscopy was performed to rule out corneal pathology.

Pre-test epithelial pachymetry using the Optovue iVue and the NIKTBUT using the Oculus Keratograph 5M were obtained. Two pachymetry readings were obtained measurements and one reading was of the average NIK-
BUT measurement being used for analysis. Measurements were conducted by the same two examiners to ensure standardisation. Each participant was then required to individually vape $0.05 \mathrm{ml}$ of e-liquid in a smokers designated area of the study site which was $4.67 \mathrm{~m}$ by $2.25 \mathrm{~m}$ with the air-conditioning turned off. Immediately after vaping the epithelial pachymetry and NIKBUT measurements were repeated to obtain post-test measurements.

\section{Ethical considerations}

Ethical clearance was obtained prior to commencement of the study from the Biomedical Research Ethics Committee (BREC) at the University of KwaZulu-Natal (RSA). Each participant was provided with an information document to inform them about the details of the study and a consent form to ensure that they were fully aware of their rights should they consent to participate in the study. Gatekeeper permission was obtained prior to the commencing the study.

\section{Results}

Sixty four participants were admitted into the study of which 43 were males and 21 were females with an average age of 21 years. Fifty eight participants had reliable pachymetry scans for corneal epithelial thickness measurements whilst fifty seven scans from topographic data were accepted for NIKBUT as reliable.

The corneal measurements were an average of two measurements whilst the first reading measurement for the average NIKBUT was analysed using Statistical Package for Social Science (SPSS) version 24. The right eyes of each participant were used for data analysis thus 58 eyes for the corneal epithelial thickness and 57 eyes for the NIKBUT measurements were analysed.

\section{Corneal epithelial thickness analysis}

Figure 1 shows for central corneal epithelial thickness a change from 52.44 microns pre-vaping to 52.76 microns post-vaping. Superior corneal epithelial thickness changed from 52.38 microns pre-vaping to 52.56 microns post-vaping. Inferior corneal epithelial thickness changed from 52.97 microns pre-vaping to 53.19 microns post-vaping. Nasal corneal epithelial thickness changed from $52.63 \mathrm{mi}-$ crons pre-vaping to 52.81 microns post-vaping. Temporal corneal epithelial thickness changed from 51.64microns pre-vaping to 51.87 microns post-vaping. All these observations were statistically insignificant at $\mathrm{p}$-values greater than 0.05 . 


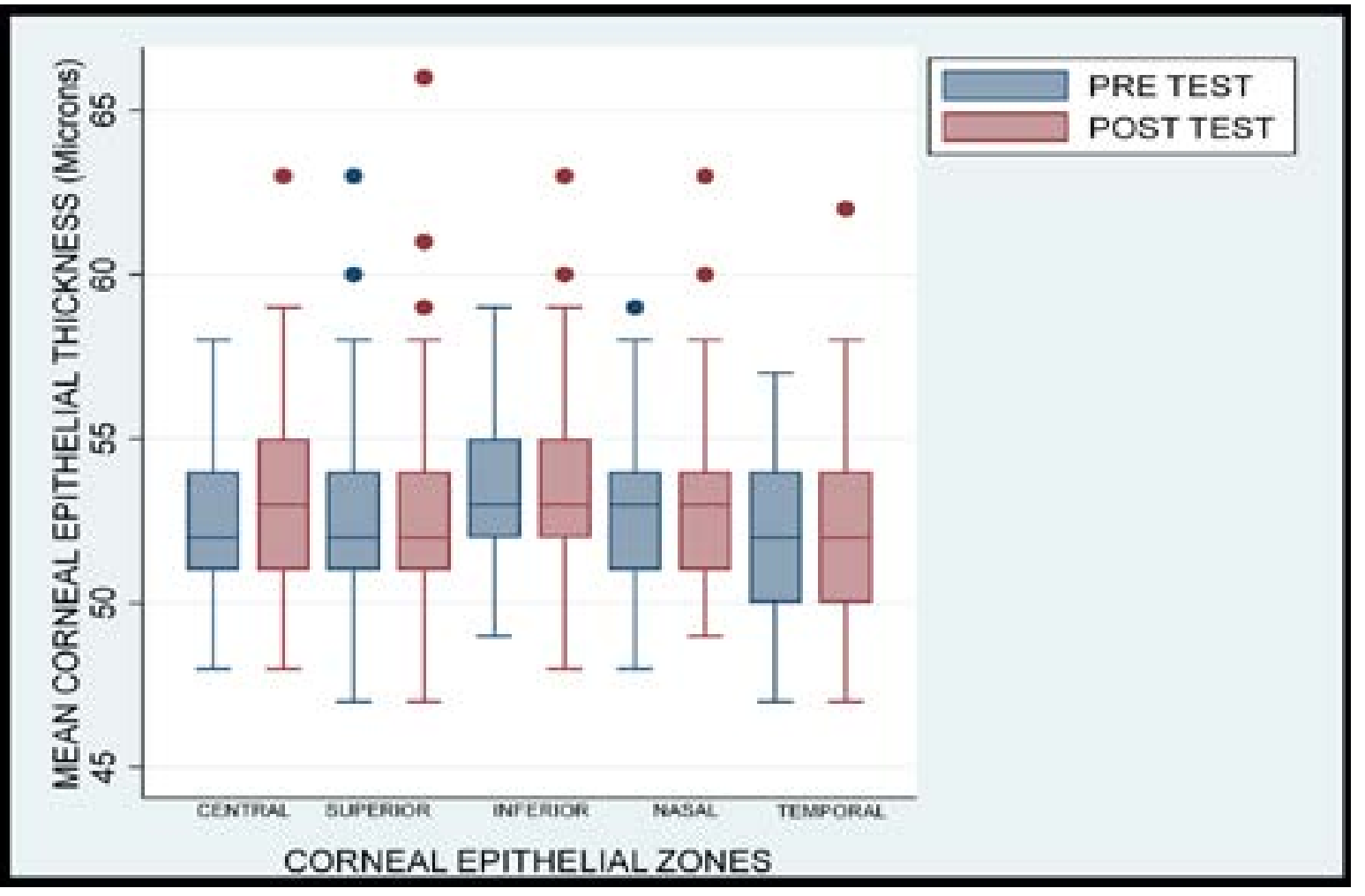

Figure 1: Box and whisker plot for mean corneal epithelial thickness before and after vaping $0.05 \mathrm{ml}$ of eliquid.

Table 1 shows the mean change for central corneal epithelial thickness was -0.3448 microns which was statistically insignificant with a p-value of 0.105 . The mean change for superior corneal epithelial thickness was $-0.2414 \mathrm{mi}-$ crons which was statistically insignificant with a p-value of 0.230 . The mean change for inferior corneal epithelial thickness was -0.2931 microns with a statistically insignificant $\mathrm{p}$-value of 0.169 . The mean change for nasal corneal epithelial thickness was -0.2069 microns with a statistically insignificant p-value of 0.269 . The mean change for temporal corneal epithelial thickness was -0.2759 microns with a statistically insignificant $\mathrm{p}$-value of 0.117 .

Table 1: NIKBUT analysis

\begin{tabular}{|c|c|c|c|c|}
\hline & $\mathbf{N}$ & Mean change & SD & P-value \\
\hline Central zone & 58 & -0.3448 & \pm 1.5955 & 0.105 \\
\hline Superior zone & 58 & -0.2414 & \pm 1.5138 & 0.230 \\
\hline Inferior zone & 58 & -0.2931 & \pm 1.6005 & 0.169 \\
\hline Nasal zone & 58 & -0.2069 & \pm 1.4112 & 0.269 \\
\hline Temporal zone & 58 & -0.2759 & \pm 1.3218 & 0.117 \\
\hline
\end{tabular}


Figure 2 shows for the sample the mean average NIKBUT changed from 12.72 seconds pre-vaping to 14.12 seconds post-vaping with a statistically insignificant $\mathrm{p}$-value of 0.089 .

Table 2 shows the mean change in average NIKBUT post-vaping was -1.40 seconds with a statistically insignificant p-value of 0.089 .

$41.1 \%$ of the participants had stable NIKBUT readings post-vaping, 42.8\% had NIKBUT readings in the intermediate range post-vaping and $16.1 \%$ had unstable NIKBUT readings post-vaping.

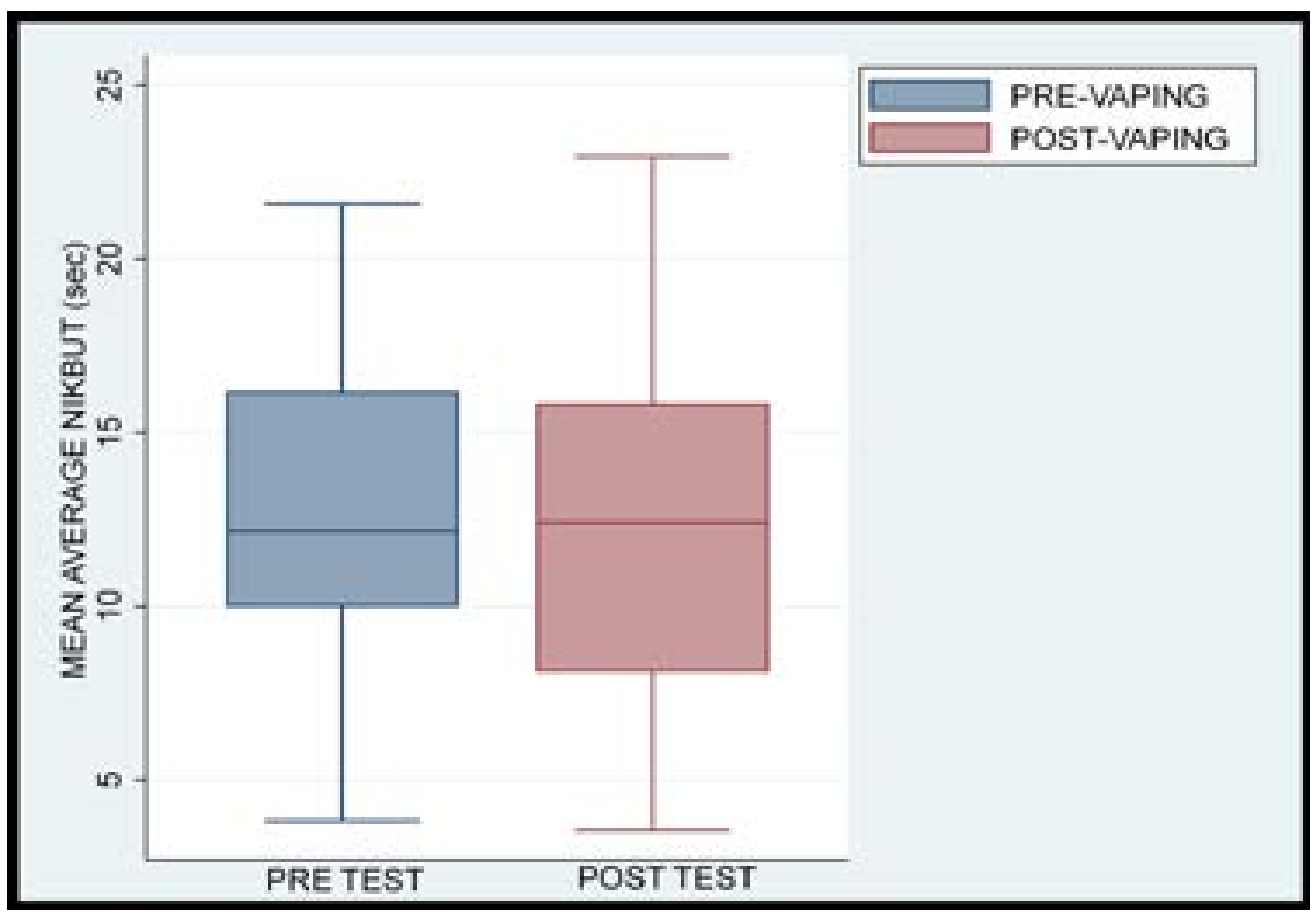

Figure 2: Box and whisker plot for Average NIKBUT before and after vaping $0.05 \mathrm{ml}$ of e- liquid.

Table 2: Mean change (microns) in average NIKBUT after vaping $0.05 \mathrm{ml}$ of e-liquid. A negative reading implies an increase in NIKBUT and a positive reading implies decrease.

\begin{tabular}{|c|c|c|c|}
\hline $\mathbf{N}$ & $\begin{array}{l}\text { Mean change S } \\
\text { (seconds) }\end{array}$ & SD & P-Value \\
\hline $\begin{array}{l}\text { PRE TEST - } 57 \\
\text { POST TEST }\end{array}$ & \begin{tabular}{l|l}
-1.40 & \pm
\end{tabular} & \pm 6.11 & 0.089 \\
\hline
\end{tabular}

\section{Discussion}

The results revealed that the specific brand of e-cigarette utilised in this study was not associated with major acute physiological changes, causing only statistically insignificant mean changes in corneal epithelial thickness in all five zones after exposure to $0.05 \mathrm{ml}$ of e-cigarette vapour, as seen in Table 1. Furthermore, despite this, comparatively the nasal zone appeared to be influenced the least and the superior zone the most. This observation is in agreement with Reinstein et $\mathrm{al}^{22}$ who observed the thicker nasal and inferior epithelial zones in normal eyes and even more so the infero-superior zones having a larger difference.

NIKBUT analysis showed a mean increase of $1.40 \mathrm{sec}-$ onds post-vaping. These findings were not in agreement with Weislander et al. ${ }^{12}$ who found that exposure to propylene glycol mist resulted in dry eye. However, the increase in NIKBUT may be attributed to the time frame between participants vaping and the measurement being recorded. The NIKBUT is a novel technique of assessing the tear film stability. In the current study, the tear stabil- 
ity was measured with NIKBUT whilst in previous studies, this was measured using tear break up time, which may account for these differences.

In contrast, several studies have shown that conventional cigarette smoking has adverse effects on the pre-corneal tear film, and that there is a strong association between smoking and tear film instability. ${ }^{23,24,15}$ Satici et al. ${ }^{23}$ reported that smoking can also cause ocular surface epithelial damage because the smoke comes into direct contact with the ocular surface. Based on this premise this study explored the effects of e-cigarettes, and may for the first time described the changes to the anterior corneal surface induced by exposure to e-cigarette vapour subject to vaping technique or other conditions such as temperature. Cigarette smoking has been reported to be one of the factors in a multitude of clinical conditions that cause ocular surface epithelial damage, reduced corneal and conjunctival sensitivity, increased superficial punctuate staining and a dysfunctional tear film, resulting in dry eye. ${ }^{15}$ Also, Jetton et $\mathrm{al}^{16}$ found a delay in corneal wound healing in smokers. In this study, we have shown that exposure to e-cigarette vapour has statistically insignificant effect on the pre-corneal tear film and corneal epithelial thickness for this novel paradigm. Intuitively, smoke should be more disruptive than vapour as smoke occurs over an open flame and is a collection of solid, liquid and gaseous particles whilst vapour is a less brutal process and is gaseous.

$0.05 \mathrm{ml}$ of e-liquid or on average 10 puffs of e-cigarette vapour may have influenced the findings. This chosen number of puffs may have been inadequate to effect changes. Furthermore, Etter et al. $^{25}$ found that on average e-cigarette user vaped 175 puffs per day. The results of Goniewicz et al. ${ }^{4}$ may have beecomplemented if there was an increase in the number of puffs. Further suggestions that the individual's puffing topography can influence the efficacy of the nicotine delivery from e-cigarette may have influenced the outcome of this study. ${ }^{4}$ Therefore future studies should attempt to standardise the puffing topography and in addition increase exposure to the daily average of number of puffs vapers vape to obtain a more realistic observation.

Nishitsuka et al. ${ }^{26}$ evaluated risk factors and determinants of corneal thickness in the Japanese population and concluded that among other factors current smoking was as- sociated with an increase in corneal thickness. The present study's findings showed a statistically insignificant change but did show a negligible amount of corneal thickening post vaping for clinical consideration. Furthermore, findings of insignificant changes may not be influenced by diurnal changes as $\mathrm{Du}$ et al. ${ }^{27}$ demonstrated the corneal epithelial thickness appears stable during the daytime.

The results demonstrated an increase in corneal epithelial thickness questioning the clinical significance of e-cigarette vaping. The influence of this on intra-ocular measurements clinically should be accounted for in e-cigarette users.

The current findings demonstrated that acute e-cigarette exposure does not have adverse effects on the pre-corneal tear film and corneal epithelial thickness. This may agree with the notion that e-cigarettes are safer than conventional cigarettes as evidenced in marketing campaigns for acute exposure but with caution. Despite the statistically insignificant changes noted in this study one should not ignore its clinical consideration. The minimal influence on corneal epithelial thickness and stable NIKBUT suggests minimal pre-op and post-op effect on refractive surgery measurements. Contact lens wearers who vape may also not be affected as the corneal epithelium is stable and the tear film does not suggest drying considering the NIKBUT results. The delayed wound healing from corneal ulcers or abrasions may not be compounded in vapers in contrast to cigarette smokers. The impact on tonometry measurements when factoring pachymetry may also stable.

Considering the results of the study showed non-significant changes, the variability of instrumentation should be discussed to account for this. Tian et $\mathrm{al}^{19}$ used the Oculus $5 \mathrm{M}$ keratograph on normal eyes and found $10.35 \pm$ $4.22 \mathrm{~s}$ for average NIKBUT, this is in agreement with the present study's pre-test average NIKBUT of $12.72 \mathrm{~s}$ and post-test average NIKBUT of 14.12 s accounting for the standard deviation. Reinstein et $\mathrm{al}^{22}$ found the central corneal epithelial thickness to be $53.4 \pm 4.6 \mu \mathrm{m}$ in normal corneas. The mean pre-test and post-test central corneal epithelial thickness was $52.44 \mu \mathrm{m}$ and $52.76 \mu \mathrm{m}$ respectively for this study, thereby accounting for the stability of data collection tools and the results obtained in this study. However, a recommendation of repeating post-test measurements a second time until pre-test measurements returned would better implicate vaping as the cause of the 
change in measurements observed. Considering the lack of changes demonstrated in the study, a control group exposed to cigarette smoke, in future research may accentuate the findings in this study.

The increase in corneal epithelial thickness in all zones post-vaping coupled with increase in NIKBUT post-vaping may suggest a clinical change that may be magnified if there was an increase in the sample size and the amount e-liquid vaped. Etter et $\mathrm{al}^{25}$ found that on average e-cigarette users vaped 175 puffs a day. The 10 puff vaping protocol was utilized as it allowed for little or no nicotine as non-smokers who volunteered for the study may not be adversely affected, however this was a limitation of the study. Thus, using vapers only and increasing the number of puffs to a representational vaping behavior may alter the finding of the study.

\section{Limitations}

A limited sample size, and a lack of access to exclusive vapers proved challenging from the study population and site. Including e-cigarette-naive subjects suggests the influence of the 'learning curve' to duplicate experienced e-cigarette users vaping technique and the amount of e-liquid may have not been significant enough to influence changes to the corneal surface. Thus, the researchers recommend the use of a larger sample size with a similar methodology including the use of non-exposed subjects under the same measuring conditions to enhance the reliability of the findings. A further recommendation of a pilot study to improve the vaping technique of novice subjects, and the measurements of corneal epithelial thickness and NIKBUT be obtained on separate occasions.

\section{Conclusion}

The study highlights the effect of vaping on anterior most corneal integrity. The results revealed that there are no statistically significant changes on pre-test post-test vaping on NIKBUT measurements. It also found that e-cigarette vaping had negligible changes on the thickness of the corneal epithelium. This infers that vaping of approximately 10 puffs has negligible changes on the epithelial structure and the stability of pre-corneal tear film.

The statistically insignificant changes in both corneal epithelial thickness and NIKBUT post-vaping suggests that vaping may not have an irritable influence on the anterior most aspect of the cornea. The researchers would like to caution that despite the statistically insignificant changes, clinical increase was noted which may be amplified with puff number and repeated vaping throughout the day. In conclusion, the findings that the corneal epithelium was not disrupted acutely post vaping is not an exhaustive conclusion.

\section{Acknowledgements}

The authors would like to thank Professor P. Brown for his guidance.

\section{Conflict of interest}

None declared.

\section{References}

1. WHO | WHO global report on trends in tobacco smoking 2000-2025. WHO. 2015.

2. DrugFacts: Electronic Cigarettes (e-Cigarettes) | National Institute on Drug Abuse (NIDA). https://www. drugabuse.gov/publications/drugfacts/electronic-cigarettes-e-cigarettes.

3. Palazzolo DL. Electronic Cigarettes and Vaping: A New Challenge in Clinical Medicine and Public Health. A Literature Review. Front Public Heal. 2013;1:56. doi:10.3389/ fpubh.2013.00056.

4. Goniewicz ML, Knysak J, Gawron M, et al. Levels of selected carcinogens and toxicants in vapour from electronic cigarettes. Tob Control. 2014;23(2):133-139. doi:10.1136/tobaccocontrol-2012-050859.

5. Hess I, Lachireddy K, Capon A. A systematic review of the health risks from passive exposure to electronic cigarette vapour. Public Heal Res Pract. 2016;26(2). doi:10.17061/phrp2621617.

6. WHO | Backgrounder on WHO report on regulation of e-cigarettes and similar products. WHO. 2015. http:// www.who.int/nmh/events/2014/backgrounder-e-cigarettes/en/. Accessed December 12, 2016.

7. MEDICINES AND RELATED SUBSTANCES ACT 101 OF 1965. Drugs Control Amend Act. 1968;29(59). http://www.hpcsa.co.za/Uploads/editor/UserFiles / downloads/legislations/acts/medicines_and_related_ sub_act_101_of_1965.pdf. Accessed March 6, 2017.

8. Meo SA, Al Asiri SA. Effects of electronic cigarette smoking on human health. Eur Rev Med Pharmacol Sci. 
2014;18(21):3315-3319. http://www.ncbi.nlm.nih.gov/ pubmed/25487945. Accessed December 12, 2016.

9. Farsalinos KE, Polosa R. Safety evaluation and risk assessment of electronic cigarettes as tobacco cigarette substitutes: a systematic review. Ther Adv Drug Saf. 2014;5(2):67-86. doi:10.1177/2042098614524430.

10. Zhang Y, Sumner W, Chen D-R. In Vitro Particle Size Distributions in Electronic and Conventional Cigarette Aerosols Suggest Comparable Deposition Patterns. Nicotine Tob Res. 2013;15(2):501-508. doi:10.1093/ntr/nts165. 11. Trtchounian A, Williams M, Talbot P. Conventional and electronic cigarettes (e-cigarettes) have different smoking characteristics. Nicotine Tob Res. 2010;12(9):905912. doi:10.1093/ntr/ntq114.

12. Wieslander G, Norbäck D, Lindgren T. Experimental exposure to propylene glycol mist in aviation emergency training: acute ocular and respiratory effects. Occup Environ Med. 2001;58(10):649-655. doi:10.1136/oem.58.10.649.

13. Altinors DD, Akça S, Akova YA, et al. Smoking Associated With Damage to the Lipid Layer of the Ocular Surface. Am J Ophthalmol. 2006;141(6):1016-1021.e1. doi:10.1016/j.ajo.2005.12.047.

14. Vardavas CI, Anagnostopoulos N, Kougias M, Evangelopoulou V, Connolly GN, Behrakis PK. Short-term Pulmonary Effects of Using an Electronic Cigarette. Chest.2012;141(6):1400-1406. doi:10.1378/chest.11-2443. 15. Thomas J, Jacob GP, Abraham L, et al. The effect of smoking on the ocular surface and the precorneal tear film. Australas Med J. 2012;5(4):221-226. doi:10.4066/ AMJ.2012.1035.

16. Jetton JA, Ding K, Kim Y, et al. Effects of Tobacco Smoking on Human Corneal Wound Healing. Cornea. 2014;33(5):453-456. doi:10.1097/ ICO.0000000000000100.

17. Su X, Wu W, Wang Y. Repeatability of corneal thickness measurement with Optovue iVue OCT and a comparative study with Pentacam and Visante OCT. Zhonghua Shiyan Yanke Zazbi/Chinese J Exp Opbthalmol. 2015;33:135140. doi:10.3760/cma.j.issn.2095-0160.2015.02.008.

18. Yeo S, Lee R, Tun Aung H, et al. Agreement of non-invasive tear break up time measurement between
Tomey RT-7000 Auto Refractor-Keratometer and Oculus Keratograph 5M. Clin Ophthalmol. 2016; Volume 10:17851790. doi:10.2147/OPTH.S110180.

19. Tian L, Qu J, zhang X, Sun X. Repeatability and Reproducibility of Noninvasive Keratograph 5M Measurements in Patients with Dry Eye Disease. J Ophthalmol. 2016; 2016:1-6. doi:10.1155/2016/8013621.

20. Zhu K, Xie W, Ying J, Yao Y. [Evaluation of tear film and meibomian gland function in dry eye patients using Keratograph 5M]. Zhejiang Da Xue Xue Bao Yi Xue Ban. 2016;45(4):422-428. http://www.ncbi.nlm.nih.gov/ pubmed/27868417. Accessed February 19, 2017.

21. Dawkins L, Corcoran O. Acute electronic cigarette use: nicotine delivery and subjective effects in regular users. Psycho Pharmacology (Berl). 2014;231(2):401-407. doi:10.1007/s00213-013-3249-8.

22. Reinstein DZ, Archer TJ, Gobbe M, et al. Epithelial thickness in the normal cornea: three-dimensional display with Artemis very high-frequency digital ultrasound. J Refract Surg. 2008;24(6):571-581. http://www.ncbi.nlm.nih. gov/pubmed/18581782. Accessed January 27, 2017.

23. Altinors DD, Akça S, Akova YA, et al. Smoking Associated With Damage to the Lipid Layer of the Ocular Surface. Am J Ophthalmol. 2006;141(6):1016-1021.e1. doi:10.1016/j.ajo.2005.12.047.

24. Matsumoto Y, Dogru M, Goto E, et al. Alterations of the tear film and ocular surface health in chronic smokers. Eye (Lond). 2008;22(7):961-968. doi:10.1038/ eye.2008.78.

25. Etter J. A longitudinal study of electronic cigarette users. Addict Behav. 2014;39(2):491-494. doi:10.1016/J. Addbeh.2013.10.028.

26. Nishitsuka K, Kawasaki R, Kanno M, et al. Determinants and Risk Factors for Central Corneal Thickness in Japanese Persons: The Funagata Study. Ophthalmic Epidemiol. 2011;18(5):244-249. doi:10.3109/09286586.2011.594206. 27. Du C, Wang J, Cui L, et al. Vertical and horizontal corneal epithelial thickness profiles determined by ultrahigh resolution optical coherence tomography. Cornea. 2012;31(9):1036-1043. doi:10.1097/ ICO.0b013e31823f8d56. 